\title{
3 Research Square

\section{Is Cell Regeneration and Infiltration a Double Edged Sword for Porcine Aortic Valve Deterioration: A Large Cohort of Histopathological Analysis}

\section{Li Li ( $D$ lili@fuwaihospital.org )}

Chinese Academy of Medical Science

\section{Xuejing Duan}

Chinese Academy of Medical Science

Hongyue Wang

Chinese Academy of Medical Science

\section{Yang Sun}

Chinese Academy of Medical Science

Wei Zhao

Chinese Academy of Medical Science

\section{Yang Lu}

Chinese Academy of Medical Science

Hongyu Xu

Chinese Academy of Medical Science

\section{Yiwei You}

Chinese Academy of Medical Science

Qingzhi Wang

Chinese Academy of Medical Science

\section{Research Article}

Keywords: Porcine aortic valve, Structure valve deterioration, Histolopathology, Valve interstitial cells regeneration, Untransformed valve endothelial cells

Posted Date: November 30th, 2021

DOI: https://doi.org/10.21203/rs.3.rs-1037827/v1

License: (c) (1) This work is licensed under a Creative Commons Attribution 4.0 International License.

Read Full License 


\section{Abstract}

Background Bioprostheses are the commonest prostheses used for valve replacement in the western world. The major flaw of bioprostheses is the occurrence of structural valve deterioration (SVD). The objective of this study was to assess in a large cohort of patients the pathologic features of porcine aortic valve (PAV) SVD based on histomorphological and immunopathological features.

Methods and materials 109 cases of resected PAV were observed grossly and histopathologically. The type and amount of infiltrated cells were evaluated in the different type of bioprosthetic SVD by immunohistochemical staining .

Results The most common cause of SVD was calcification, leaflet dehiscence and tear $(23.9 \%, 19.3 \%$ and $18.3 \%$, respectively). Immunohistochemical staining demonstrated that vimentin positive cells aggregated around the calcified area in calcified PAV. Macrophages infiltrated in the calcified, lacerated and dehiscence PAV. However, MMP-1 expression was mainly found in the lacerated PAV. The VIM(+)/SMA(-) and VIM(+)/CD31(-) cells were found in PAV. The endothelia rate of dehiscence leaflets were higher than that of calcified and lacerated leaflets. A large amount of CD31 positive cells aggregated in the spongy layer in the lacerated and dehiscence PAV.

Conclusions Cell regeneration and infiltration is a double edged sword for the PAV deterioration. Valve interstitial cells (VIC) have essential role in PAV calcification. Macrophages infiltration maybe involve in the different type of SVD, but only MMP-1expression involves in leaflets laceration. VIM(+)/CD31(-) valve endothelial cells (VECs) protect the PAV against the formation of calcified and lacerated lesions. The existence of untransformed VECs maybe one of pathologic substrate of PAV tear and dehiscence, although they can prevent VICs activation and subsequent valve fibrosis and calcification.

\section{Background}

Valve replacement is a common procedure in the treatment of valvular diseases, and there are about 200,000 cases worldwide. Especially in the last decade, under the influence of the aging trend of the population, coupled with the continuous improvement of the hemodynamic performance of commercial biological prostheses [1], biological prostheses are more inclined to replace mechanical prostheses in the selection of prostheses category. After the verification of long-term application process, bioprosthetic valve underwent structural deterioration including calcification, valve orifice narrowing and tearing of leaflets [2]. The process of crosslinking with glutaraldehyde has attracted widespread attention [3], and various methods have been developed by valve companies to ensure that this calcification process can be effectively avoided [4]. However, the process of tearing and dehiscence of leaflets has been neglected. The porcine aortic valve (PAV) tear or dehiscence is more prevalent than bovine pericardial valve (BPV) in our previous study[5]. Traditionally, structural valve deterioration (SVD) diagnosis often has been based on echocardiography and surgical observation, leading to incompletely understand the mechanism of 
SVD. The present study aims to analyses the pathological features of PAV SVD especially leaflets tear and dehiscence based on histomorphology and immunopathology in a large cohort of patients.

It is considered that bioprostheses undergo SVD due to the lack of a regenerative capability upon chemical fixation and decellular processes [6-8]. However, it has been observed in recent years that native valve calcification and myxomatous is an active process. The inflammatory cells, valvular interstitial cells (VICs) and valvular endothelial cells (VECs) are all involved in an active process [9-11]. To sum up, based on these findings, the focus of this study is to explore the role of active biological processes in porcine aortic valve SVD and the prevention measures of leaflets tearing and dehiscence.

\section{Methods And Materials}

\section{Patients}

In order to conduct a retrospective study of this procedure, we conducted a systematic induction and analysis of the age and other information of all subjects who underwent porcine aortic valve replacement in our institution from1st January 2006 to 31 December 2020. This study was approved by the Institutional Review Board at Fuwai Hospital. Informed consent was obtained from all patients and investigation were conducted per the guidelines of the Declaration of Helsinki principles.

\section{Pathological observation}

All PAVs fixed in buffered Formalin were observed grossly, dehydrated and wrapped in paraffin. Slides with 4-5 $\mu \mathrm{m}$ thickness were sectioned, deparaffinized, and stained with H\&E and Elastic staining. The histological feature was observed by Zeiss microscopy including the collagen and elastic fibers disruption, collagen delamination and plasma protein permeation,etc. The mode of bioprosthetic valve failure were recorded according to the recommendation [6] (1) Structural dysfunction: intrinsic permanent valve alterations that lead to hemodynamic or clinical dysfunction, which include calcification, leaflet tear, dehiscence, wear, fracture, poppet escape, embolization. (2) Nonstructural dysfunction can lead to changes in hemodynamics such as pannus, suture entrapment, parvalvular leakage and proportional imbalance that are nonintrinsic. (3) Thrombosis: development of thrombus on any component of valve. (4) Endocarditis: infection of any valve structure.

\section{Quantification of collagen}

The density of type I and III collagen in valve tissue slices was measured using picro-sirius red staining and then observed with polarizing microscopy as previously reported [12]. We measured the ratio of type I collagen to type III collagen by digital planar measurement method, and the collagen density was calculated by dividing the calculated area of type I collagen by the area of type III collagen. 


\section{Immunohistochemistry stainings}

Immunohistochemical staining was performed in calcified, dehiscence, tear and non SVD PAV with 10 cases in each group. The valve tissue section $(5 \mathrm{~mm})$ was incubated with specific primary antibody including rabbit monoclonal antibody to smooth muscle actin, vimontin, CD68, MMP-1 diluted at the ratio of $1: 100,1: 250,1: 100,1: 100$ ), rabbit polyclonal antibody to CD34, CD31 diluted at the ratio of 1:2500 (Cell Signaling Technology, Inc, CA). Then the sections were incubated with the IHC Detection Reagent (HRP, Rabbit. Cell Signaling Technology, Inc, CA) as described by the manufacturer's instruction. At the last the sections were developed with a diaminobenzidine kit (DAB kit; Thermo Fisher Scientific Inc. MA, USA), and counter-stained with weak hematoxylin. The number of infiltrating cells in the bioprosthetic valve region was semi-quantified based on the number of inflammatory cells per high-magnification field and distribution of inflammation[13]. The endothelium rate was calculated by dividing the endothelium area by the total area of leaflet surface.

\section{Statistical analysis}

The data were expressed by means \pm SEM. By comparing the two groups of data, the two-tail Student $T$ test and Levene $\mathrm{F}$ test mann-Whitney nonparametric test were used to compare the Gaussian distribution samples and the non-Gaussian distribution samples with equal variance. One-way ANOVA focuses on multiple comparisons. Percentages of different types of SVD in mitral and aortic bioprostheses were compared using cross-stabbing. The analysis variables included type I/III collagen ratio and the infiltrated cell grade.

\section{Results}

\section{Clinical features}

In Fuwai hospital, the number of valve replacements was approximately 3000 cases per year and $17.7 \%$ of prostheses was bioprosthetic valve. A total 143 cases of bioprostheses were collected including 109 PAV and 34 BPV. 109 PAVs were involved in the study with the average age of patients of $56.77 \pm 17.40$ years old (range from $5-78 \mathrm{yrs}$ ) including $36.7 \%$ of male and $63.3 \%$ of female. The life span was $8.74 \pm 3.75$ years $(0.4-18 \mathrm{yrs})$. There was no difference of the life span between the mitral bioprosthetic valve and the aortic bioprosthestic valve $(8.78 \pm 3.75$ vs $8.69 \pm 4.48$ years, $p=0.909)$.

\section{Mode of PAV failure}

These 109 PAVs included 65 mitral valves, 40 aortic valves and 4 tricuspids. The most common cause of PAV failure was calcification $(23.9 \%, 29 / 109)$, followed by leaflets dehiscence $(19.3 \%, 21 / 109)$, tear (18.3\%,20/109), infective endocarditis $(10.1 \%, 10 / 109)$, pannus $(9.2 \%, 12 / 109)$, calcification accompanied with leaflets tear or dehiscence $(4.6 \%, 4 / 109)$, thrombus $(4.6 \%, 4 / 109)$, accompanied surgery $(6.4 \%, 7 / 109)$, paravalvular leak $(0.9 \%, 1 / 109)$. The percentage of mitral PAV leaflet dehiscence was higher 
than aortic PAV $\left(30.8 \%\right.$ vs $\left.5.0 \%, \chi^{2}=9.928, p=0.001\right)$. Statistically, there was no significant difference in the rate of mitral and aortic valve tear or calcification $\left(20.0 \%\right.$ vs $17.5 \%, \chi^{2}=0.100, P=0.751$ and $33.8 \%$ vs $\left.20.0 \%, \chi^{2}=2.326, P=0.127\right)$.

\section{Histological findings of PAV with calcification, tear and dehiscence}

Leaflets are presented in an intact structure in non SVD PAV (pannus). Type I collagen, elastic fibers and type III collagen are located in dense layer, ventricular layer, ventricular layer and sponge layer respectively, similar to PAV in normal state (Figure 1a). The lacerated leaflets exhibited collagen delamination or abrupt into debris and elastic fiber also showed breaking or disappear (Figure 1b). Plasma protein permeating was observed. By picro-sirius red staining and observed by double refraction microscopy, Collagen I which showed a red-yellow double refraction in dense layer and ventricular layer showed delaminated and fractured into debris, more collagen III which showed a red-green double refraction were found in all three layers of the lacerated leaflets. Collagen delamination generally occurs in the hinge region of the dehiscence PAV leaflets. Collagen III increase was also observed, although the degree was milder than in the lacerated PAV. In most calcified PAV, the leaflets showed dense collagen deposition in dense layer. Compared with PAV with leaflets tear and dehiscence, type I collagen increased around the calcified area in calcified PAV, and the ratio of type I collagen to type III collagen was higher( $8.35 \pm 6.91$ vs $1.09 \pm 0.37$ and $0.66 \pm 0.19, p=0.016$ and 0.044 ) (Figure 1c). There was no difference of collagen I to III ratio between PAV with leaflets tear and dehiscence $(1.09 \pm 0.37$ vs $0.66 \pm 0.19, p=0.902)$.

\section{Cell regeneration/infiltration in PAV with calcification, tear and dehiscence}

Immunohistochemical staining revealed an aggregation of vimentin (VIM) positive cells in calcified, lacerated and dehiscence PAV (Figure 2a). Some vimentin positive cells are also CD68 positive, which indicates that macrophages exist objectively. However, these cells can not express smooth muscle actin (SMA). CD68 (-)/VIM (+) cells were mainly distributed around the calcified area. Monolayer VIM (+) cells mainly attached to the surface of leaflets, which showed the morphology of endothelial cells, but the expression of CD31 was negative. The endothelia rate of dehiscence leaflets were higher than that of calcified and lacerated leaflets $(54.00 \pm 27.01 \%$ vs $25.20 \pm 23.24 \%$ and $23.91 \pm 25.82 \%, p=0.033$ and 0.051 , respectively), which indicated the protective role of endothelia against the leaflets calcified and lacerated lesion. The grade of vimentin expression was higher in the calcified PAV, compared with lacerated and dehiscence leaflets $(2.20 \pm 0.86$ vs $1.11 \pm 1.06$ and $1.14 \pm 1.07, p=0.011$ and 0.022 , respectively). As indicated by the CD68, PAV exhibited a significant macrophage infiltration in the calcified, lacerated and dehiscence leaflets, especially in the delaminated or calcified area (Figure 2b). There was no difference among the grade of macrophages in the different groups. MMP-1 was expressed in lacerated leaflets, and the expression level was higher than that in dehiscence leaflets $(1.11 \pm 0.98$ vs $0.17 \pm 0.40, p=0.037)$ (Figure 2c). A large amount of CD31 positive cells aggregated in the spongy layer of the lacerated and dehiscence PAV but not calcified and non SVD PAV (Figure 2d). The grade of infiltrated CD31(+) cells was 
higher in the lacerated and dehiscence leaflets compared to the calcified leaflets $(2.17 \pm 1.19$ and $2.60 \pm 0.89$ vs $0.83 \pm 0.94, p=0.006$ and 0.003 respectively). CD68, CD34, vimentin and SMA were negative in these cells. As a control, the normal porcine aortic valve demonstrated that a few valve interstitial cells (VICs) located in all three layer which showed vimentin and SMA coexpression (Figure 2e).

\section{Discussion}

It is considered that the bioprosthetic valve dehiscence is due to high-speed blood shear stress. The evidence is that mitral valve is more easier to occur dehiscence than aortic valve because of higher blood flow impact which is demonstrated in the present study and other studies $[5,6,9]$. However, there has been a paucity of publications to associate the changes of valve histological structure with dehiscence in PAV. Mao, et al found that the microstructural damages occurred in pericardial patch during manufacturing process may identify the vulnerable sites that play an important role in the cusp dehiscence of bovine pericardial valve. As an important part of pericardial patch, manufacturing process will cause the elimination of collagen bundle ripple or layered damage around the scaffold [14]. The above research shows that there will be corresponding changes at the suture around the stent. Compared with the single package design, the connecting area of three separate tips is larger. In my opinion, the key factors causing the relative concentration of stress mainly include: uneven or mismatched stent, suture hole and joint [14]. In addition, it was also found that macrophages had invaded the cracked area and produced MMP-1 expression, although the expression of MMP-1 was relatively mild relative to the lacerated leaflets. In the pathogenesis of calcified aortic stenosis, MMP, an extracellular matrix protein, is the most common [15]. MMPs can perform pathological remodeling in multiple tissues and in the remodeling extracellular matrix [16]. The local balance between MMPs and TIMPs plays a decisive role in tissue remodeling $[16,17]$. Among them, MMP-1 comes from macrophages and fibroblasts. In terms of affinity, collagen fibers are the most obvious. It is conducive to activating type I collagen decomposition [18]. Few studies have been performed to investigate the MMP-1 in the SVD PAV. Our study show that MMP-1 expression is mainly expressed by macrophages in the lacerated PAV and the damage area in the dehiscence PAV. Most previous studies focus on the MMP-1 and native valve calcification and showed a correlation of MMP-1 level with valve stenosis[16,19,20]. Different from their studies, we do not find the increased MMP-1 expression in calcified PAV, although the macrophage infiltration is obvious. This divergence demonstrate that the mechanism of bioprostheses calcification is not the MMP-1 dependent. Other MMPs family members involved should be investigated in the future. How to decrease the suture area in the bioprostheses and anti-inflammatory therapy would be the effective methods to alleviate the dehiscence in PAV.

Differing from dehiscence, lacerated leaflets showed the overall valve structure damage including collagen disrupt into debris and plasma protein permeation as demonstrated in the present study. Increased type III collagen content and decreased ratio of collagen I/III were also found in the lacerated leaflets. The collagen matrix of normal aortic valves is mainly formed by type I collagen (70\%), with about $25 \%$ of type III[21]. Purushothaman et al. [22] proved that increasing the density of type III collagen and proteoglycan on the basis of reducing the density of type I collagen will cause the expansion of sponge 
layer and affect the function of mitral valve. It was found that low type I collagen density would damage the formation of thick functional type I collagen fibers. However, type III collagen has better ductility than thick type I collagen. The intuitive response to injury is manifested in the synthesis of type III collagen [23].

It was believed that the PAV calcification was the consequence of damaged cell regeneration and selfrepairing after injury. However, in the present study, VICs are found aggregated around the calcified area in PAV which demonstrate their essential role in the bioprostheses calcification. Valve interstitial cells are the most abundant cell types in the native valves and are distributed throughout all three layers [24]. Quite a number of studies focus on the function of VICs in native valvular calcification and myxomatous degeneration $[25,26,27]$. VICs have the ability of differentiating into myofibroblast-like VICs or osteoblastlike VICs under the pathogenic condition and promote heart valve calcification and ossification. Sanger et al. found that healthy quiescent mitral VICs undergo a phenotypic activation via up regulation of Bone Morphogenetic Protein 4 (BMP4)-mediated pathway and promote mitral valve myxomatous degeneration[9]. In native valve, endothelial-to-mesenchymal transition (EndMT) has been shown to play a role in the pathogenesis of many cardiovascular diseases including heart valve disease[28,29]. In this transition, VECs acquire the phenotypic and functional characteristics of VICs and express both endothelial and mesenchymal cell markers[30]. Although several studies had supports the existence of EndMT process both in vivo and in vitro in calcified aortic valve disease [31,32], this has not been confirmed in the calcific bioprostheses. In the present study, we do not find the cell with both VECs and VICs biomarker. So the origin of VICs in the calcific bioprostheses is still a unresolved question.

The interesting finding in the present study is the existence of $\mathrm{VIM}(+) / \mathrm{SMA}(-)$ cells and the latter is one of the biomarkers of VICs in native heart valve. At the same time, we also find the VIM(+)/CD31(-) cells located on the surface of PAV. These findings indicate that the VICs and VECs regenerated in the bioprostheses are immature cells. It is reported that VIC and VEC derived from human pluripotent stem cells (hPSCs) controlled by several pathway including BMP, FGF, and Wnt ,etc[33-35]. Jiao et al. found that hPSCs with truncated Notch1 have impaired smooth muscle cell and endothelial differentiation which showed the decreased expression of SMA and CD31[35]. Relevant studies have shown that the formation of thoracic aortic aneurysm in some patients may be caused by Notch1 gene mutation affecting the SMC differentiation of bicuspid aortic valve [35]. There has been a paucity of studies focus on the mechanism and role of immature VICs and VECs in PAV. So further investigation on the pathways of the derivation of VICs and VECs need to be performed. Whereas, these immature VICs and VECs still have their essential functions including inducing bioprostheses calcification by VICs pathologically differentiate into myofibroblast-like VICs or osteoblast-like VICs. In addition, relevant studies have found that valve calcification lesions can also be protected and prevented by vascular endothelial cells[36]. Rechards et al. found that nitric oxide synthase (NOS) III expression of VECs on both sides of the calcified valves was significantly reduced, compared to normal valves. Valve endothelial-derived NO is a natural inhibitor of valve calcification and therefore may be an important protector for valve calcification[36]. We think that the ability of endothelial regeneration would provide the insight to slow down the progress of bioprostheses calcification. 
It was found that a large number of CD31 positive cells existed in the sponge layer of PAV. The role and origin of these cells are unknown. Farray reported one group VECs resistant to EndMT stimulated by TNFa. These untransformed cells expressed stabilized levels of the endothelial markers eNOS and VEcadherin but not SMA[37]. In accordance with Farray 's study, these CD31(+) cells showed SMA and vimentin negative expression and had the character of untransformed VECs in the present study. The role of these untransformed VECs in PAV tear or dehiscence is still uncovered. Farray et al. Also found that the level of Notch1 in untransformed VECs was lower than that in control VECs, which means that the EndMT potential or resistance to EndMT may be reduced[37]. In non transformed vascular endothelial cells, BMP4 will show a downward state, which is highly consistent with the state of cells in valve disease. When VEC is not transformed, inflammatory adhesion molecules ICAM-1 and VCAM- 1 still show continuous activation, which indicates that EndMT will not affect the VEC population resistant to transformation to maintain the inflammatory phenotype in TNFa treatment. These characteristics suggest that this population of cells may possess a distinct molecular signature which protects against some of the detrimental effects of inflammation[37]. In the presents study, we supposed that the untransformed VECs have both protective and detrimental effects in the leaflet laceration. The resistance of EndMT of these cells can prevent VICs activation and subsequent valve fibrosis and calcification. In addition, the nontransformed VEC will attract inflammatory cells and secrete an MMP to destroy the extracellular matrix. And bioprosthetic valve can not self-repair by secreting collagen and other extracellular matrix without VICs activation by EndMT.

There were certain limitations to our experimental design. First, we did not analyze the characteristics of different brands of commercial bioprosthetic valves because of the limited sample size. There must be dissimilarity in the histological feature between these different brands. It is necessary to collect the more bioprosthetic valves to analyze respectively. In addition, in the present study, we found CD31 positive cells in the tear and dehiscence PAV and deduced that these cells were untransformed VECs referring to the previous studies. More works must be performed to indentify these cells including more immunohistochemical stainings and single-cell RNA sequencing. Furthermore, the functions of these cells on the pathogenesis of SVD should be investigate in the future.

\section{Conclusions}

Through the investigation of a series of cases of structural valve deterioration, the histopathological characteristics of porcine aortic valve were deeply analyzed. We have shown the changes of collagen type and density and macrophages infiltration accompanied by MMP-1 expression are the major histologic markers and pathogenesis of PAV valve tear and dehiscence. Meanwhile, immature VICs and VECs are found in the acellular PAV which have different immunophenotype but similar role of mature VICs and VECs. We also find the untransformed VECs in dehiscence and tear PAV, which maybe one of pathologic substrate of PAV tear and dehiscence, although they can prevent VICs activation and subsequent valve fibrosis and calcification. 


\section{Abbreviations}

Structural valve deterioration SVD

Porcine aortic valve PAV

Valve interstitial cells VICs_

Valve endothelial cells VECs

Bone Morphogenetic Protein 4 BMP4

Metal matrix protease 1 MMP-1

Tissue inhibitors of metalloproteinases TIMPs

human pluripotent stem cells hPSCs

Nitric oxide synthase NOS

Endothelial-to-mesenchymal transition EndMT

\section{Declarations}

\section{Ethics approval and consent to participate:}

This study was approved by the Institutional Review Board at Fuwai Hospital. Informed consent was obtained from all patients and investigation were conducted per the guidelines of the Declaration of Helsinki principles.

\section{Consent for publication:}

Not applicable.

\section{Competing interests:}

The authors declare that they have no competing interests.

\section{Availability of Data and Materials:}


All data generated or analysed during this study are included in this published article. My data do not include "DNA and RNA sequences, Genomics and transcriptomics datasets, Linked phenotype and genotype data for human subjects and Gene expression data".

\section{Funding:}

No funding was obtained for this study

\section{Author details:}

1. Department of pathology, Fuwai Hospital, Peking Union Medical College \& Chinese Academy of Medical Sciences, No. 167, Beilishi Road, Xicheng District, Beijing 100037, China.

2.Center for adult surgeon, Fuwai Hospital, Peking Union Medical College \& Chinese Academy of Medical Sciences, No. 167, Beilishi Road, Xicheng District, Beijing 100037, China.

\section{Authors' contributions:}

LL designed the work and drafted the manuscript; XJD and YS analyzed data; HYW substantively revised the manuscript; WZ: analyzed and interpreted the patient data; $Y L$ and HYX performed the immunohistochemistry; YWY and QZW performed H\&e and elastic staining. All authors have read and approved the manuscript.

\section{Acknowledgements:}

Not applicable

\section{References}

1. Zhu A S and Grande-Allen KJ. Heart valve tissue engineering for valve replacement and disease modeling. Curr Opin Biomed Eng 2018; 5:35-41.

2. Le Tourneau T, Savoye C, McFadden EP, Grandmougin D, Carton HF, et al. Mid-term comparative follow-up after aortic valve replacement with Carpentier-Edwards and Pericarbon pericardial prostheses. Circulation. 1999;100(19 suppl):II11-Il16.

3. Yavahare NR, Jones PL, Hirsch D, Schoen FJ, Levy RJ. Prevention of glutaraldehyde-fixed bioprosthetic valve calcification by alcohol pretreatment, further mechanistic studies. J Heart Valve 2009;9:561-6

4. Thiene $G$ and Valente $M$. Anticalcification strategies to increase bioprosthetic valve durability. J Heart Valve Dis 2011;20:37-44 
5. Duan XJ, Wang HY, Xu JP, Li L, Xu HY, Wang QZ. Surgical pathology analysis of the causes of failure of 48 bioprosthetic heart valve in 40 Chinese cases. Zhonghua Wai Ke Za Zhi 2016;1:710-715

6. Capodanno D, Petronio AS, Prendergast B, Eltchaninoff H, Vahanian A, et al. Standardized definitions of structural deterioration and valve failure in assessing long-term durability of transcatheter and surgical aortic bioprosthetic valves: A consensus statement from the European Association of Percutaneous Cardiovascular Interventions (EAPCI) endorsed by the European Society of Cardiology (ESC) and the European Association for Cardio-Thoracic Surgery (EACTS). Eur Heart J 2017; 38:3382-3390.

7. Dvir D, Bourguignon T, Otto CM, Hahn RT, Rosenhek R, et al. VIVID (Valve in Valve International Data) Investigators. Standardized definition of structural valve degeneration for surgical and transcatheter bioprosthetic aortic valves. Circulation 2018; 137: 388-399.

8. Manji RA, Lee W, Cooper DKC. Xenograft bioprosthetic heart valves: past, present and future. Int J Surg 2015;23:280-284.

9. Sainger R, Grau JB, Branchetti E, Poggio P, Seefried WF, et al. Human myxomatous mitral valve prolapse: role of bone morphogenetic protein 4 in valvular interstitial cell activation. J Cell Physiol 2012,227:2595-2604.

10. Rutkovskiy A, Malashicheva A, Sullivan G, Bogdanova M, Stenslokken AKO, et al. Valve interstitial cells: the key to understand of heart valve calcification. J Am Heart Assoc 2017;14:e006339.

11. Xu K, Xie S, Huang Y, Zhou T, Liu M, et al. Cell-type transcriptome atlas of human aortic valves reveal cell heterogeneity and endothelial to mesenchymal transition involved in calcific aortic valve disease. Arterioscler Thromb Vasc Biol 2020;40:2910-2921.

12. Bourgine PE, Pippenger BE, Todorov A Jr, Tchang L, Martin I. Tissue decellularization by activation of programmed cell death. Biomaterials. 2013;34:6099-6108.

13. Yahagi K, Ladich E, Kutys R, Mori H, Svensson L, et al. Pathology of ballon-expandable transcatheter aortic valves. Catheter Cardiovasc Interv 2017 15;90:1048-1057

14. Mao J, Wang Y, Philippe E, Cianciulli T, Vesely I, et al. Microstructural alterations owing to handling of bovine pericardium to manufacture bioprosthetic heart valves: A potential risk for cusp dehiscence. Morphologie 2017; 101:77-87

15. Mittal R, Patel AP, Debs LH, Nguyen D, Patel K, Grati M, et al. Intricate functions of matrix metalloproteinases in physiological and pathological conditions. Journal of cellular physiology. 2016; 31:2599-2621.

16. Helske S, Syväranta S, Lindstedt KA, Lappalainen J, Oörni K, et al. Increased expression of elastolytic cathepsins $\mathrm{S}, \mathrm{K}$, and $\mathrm{V}$ and their inhibitor cystatin $\mathrm{C}$ in stenotic aortic valves. Arterioscler Thromb Vasc Biol 2006; 26:1791-1798.

17. Bossé Y, Miqdad A, Fournier D, Pépin A, Pibarot P, Mathieu P. Refining molecular pathways leading to calcific aortic valve stenosis by studying gene expression profile of normal and calcified stenotic human aortic valves. Circ. Cardiovasc. Genet 2009; 2:489-498

18. Lurins J, Lurina D, Svirskes S, Nora-Krukle A, Tretjakovs P, et al. Impact of several proinflammatory and cell degradation factors in patients with aortic valve stenosis. Exp Ther Med 2019;17:2433-2442. 
19. Banerjee T, MukherjeeS, Ghosh S, Niswas M, Dutta S, et al. Clinical Significance of Markers of Collagen Metabolism in Rheumatic Mitral Valve Disease. PLoS One 2014;9:e90257

20. Sacks SMS and Yoganathan AP. Heart valve function: a biomechanical perspective. Philos Trans R Soc Lond B Biol Sci. 2007; 29:362:1369-1391

21. Schoen FJ. Aortic valve structure-function correlations: Role of elastic fibers no longer a stretch of the imagination. J Heart Valve Dis 1997; 6: 1-6.

22. Purushothaman KR, Purushothaman M, Turnbull IC, Adams DH, Anyanwu A, et al. Association of altered collagen content and lysyl oxidase expression in degenerative mitral valve disease. Cardiovas Pathol, 2017;29:11-18

23. Doillon CJ, Dunn MG, Bender E, Silver FH. Collagen fiber formation in repair tissue: development of strength and toughness. Coll Relat Res 1985; 5:481-592.

24. Prunotto M, Caimmi PP, Bongiovanni M. Cellular pathology of mitral valve prolapse. Cardiovas Pathol 2010;19:e113-e117.

25. Galeone A, Brunetti G, Oranger A, Greco G, Di Benedetto A, et al. Aortic valvular interstitial cells apoptosis and calcification are mediated by TNF-related apoptosis-inducing ligand. Int $\mathrm{J}$ Cardiol 2013; 169: 296-304.

26. Sun C, Liu H, Si K, Wu Y, Zhao K, et al. Meis2 represses the osteoblastic transdifferentiation of aortic valve interstitial cells through the Notch1/Twist1 pathway. Biochem Biophys Res Commun 2019; 509: 455-461.

27. Alsan BH and Schultheiss TM. Regulation of avian cardiogenesis by Fgf8 signaling. Development 2002; 129: 1935-1943

28. Zeisberg EM, Tarnavski O, Zeisberg M, Dorfman AL, McMullen JR, et al. Endothelial-to-mesenchymal transition contributes to cardiac fbrosis. Nat Med. 2007;13:952-961.

29. Anbara T, Sharif M, Aboutaleb N. Endothelial to mesenchymal transition in the cardiogenesis and cardiovascular diseases. Curr Cardiol Rev 2020;16:306-314.

30. Yu W, Liu Z, An S, Zhao J, Xiao L, et al. The endothelial-mesenchymal transition (EndMT) and tissue regeneration. Curr Stem Cell Res Ther 2014; 9:196-204

31. Perez L, Munoz-Durango N, Riedel CA, Echeverria C, Kalergis AM, et al. Endothelial-to-mesenchymal transition: cytokine mediated pathways that determine endothelial fibrosis under inflammatory conditions. Cytokine Growth Factor Rev 2017; 33:41-54.

32. Dejana E, Hirschi KK, Simons M. The molecular basis of endothelial cell plasticity. Nat Commun 2017; 8:14361.

33. Barron M, Gao M, Lough J. Requirement for BMP and FGF signaling during cardiogenic induction in non-precardiac mesoderm is specific, transient, and cooperative. Dev Dyn 2000;218:383-393.

34. van Wijk B, Moorman AF, van den Hoff MJ. Role of bone morphogenetic proteins in cardiac differentiation. Cardiovasc Res 2007;74:244-255.

35. Jiao J, Weihua T, Ping Q, Elizabeth LN, Michael MW, et al. Induced pluripotent stem cells with NOTCH1 gene mutation show impaired differentiation into smooth muscle and endothelial cells: Implications for bicuspid aortic valve-related aortopathy. J Thorac Cardiovasc Surg 2018; 156:515522. 
36. Richards J, El-Hamamsy I, Chen S, Sarang Z, Sarathchandra P, et al. Side-specific endothelial dependent regulation of aortic valve calcification: interplay of hemodynamics and nitric oxide signaling. Am J Pathol 2013; 182:1922-1931

37. Farray EJ and Butcher JT. Heterogeneous Susceptibility of Valve Endothelial Cells to Mesenchymal transformation in response to TNF-a. Ann Biomed Engineering 2014;42:149-161.

\section{Figures}
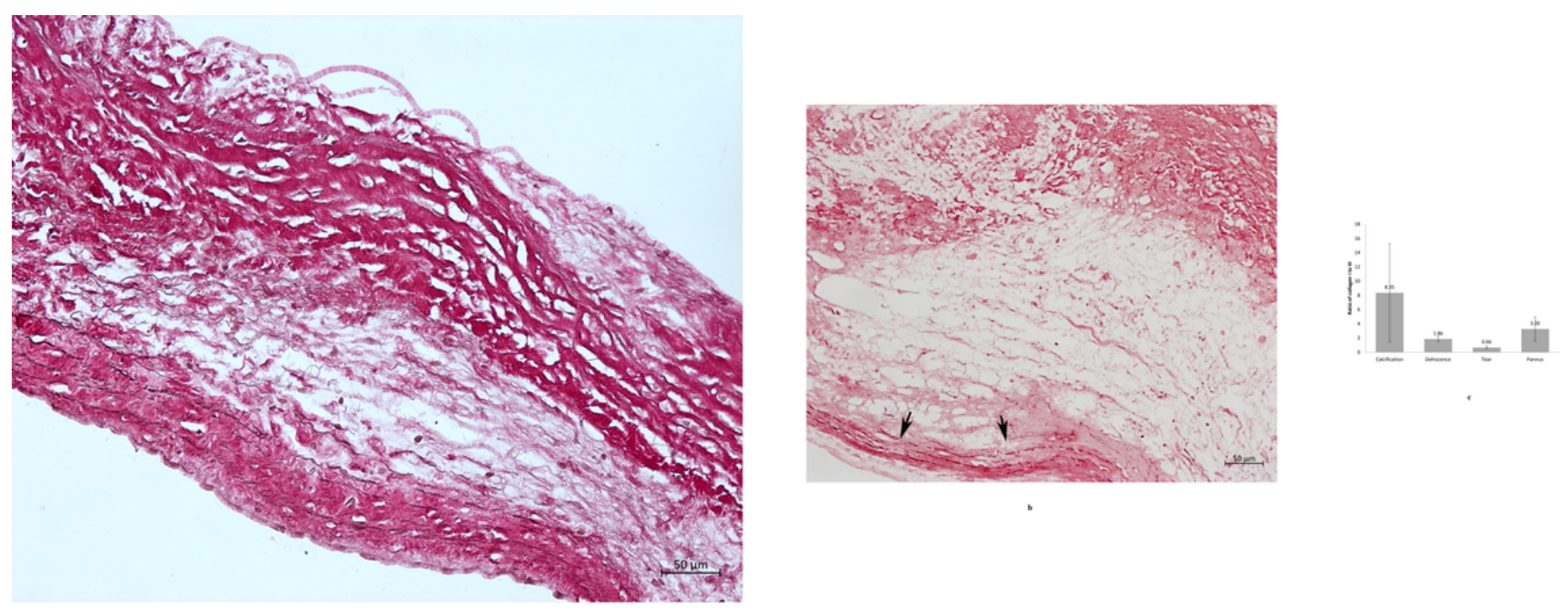

\section{Figure 1}

Collagen and elastic fiber density and arrangement in different types of PAV failure. a, Normal PAV showed intact leaflet structure with collagen I located in dense layer and ventricular layer. Elastic fiber was located in ventricular layer in normal PAV (Elastic staining, original magnification 200ロ). b, Elastic fiber broke into debris in lacerated leaflets (Arrow showed, elastic staining, original magnification 200ロ). c, The ratio of collagen I to III was the lowest in the lacerated PAV compared to the calcification PAV (* $\mathrm{P}<0.05$, compared with calcification).
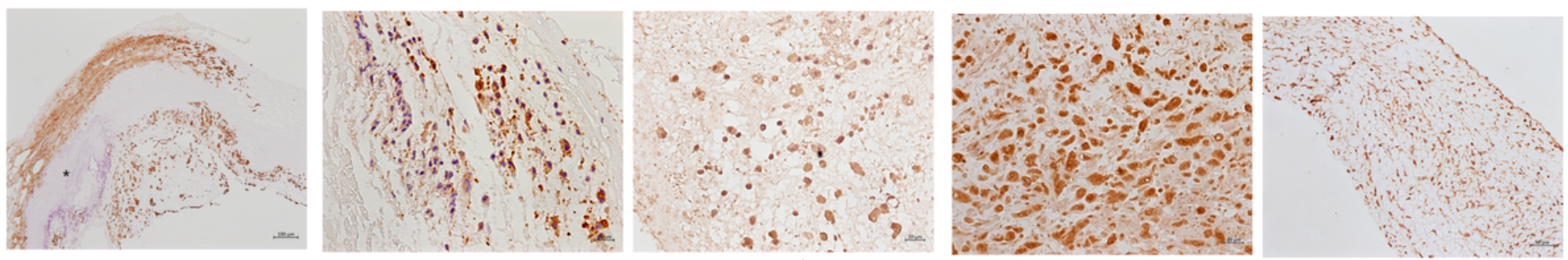

\section{Figure 2}

Cell regeneration and infiltration in different types of PAV deterioration. a, VICs expressed vimentin and aggregated around the calcified area(*) in the calcified PAV (Vimentin, original magnification, 200'); b, 
CD68 positive macrophages infiltrated in the damage area of PAV and expressed MMP-1(c) (Original magnification, 200'); d, CD31 positive cells aggregated in the spongy layer of the lacerated leaflets (Original magnification, 200'); e, In normal PAV, VICs were located in three layers with vimentin expression (Original magnification, 200'). 